COPYRIGHT @ 2019 INTERNATIONAL JOURNAL OF SCIENCE DENTISTRY | AVAILABLE ONLINE

http://www.periodicos.uff.br/index

\title{
ENXERTO DO CORPO ADIPOSO BUCAL PARA FECHAMENTO DE FÍSTULA BUCO-SINUSAL: REVISÃO DE LITERATURA
}

\section{USE OF BODY ADIPOSAL BODY WIPER FOR OROANTRAL FISTULA CLOSURE: LITERATURE REVIEW}

\section{Farley Souza Cunha}

Cirurgião-dentista graduado pela UFF e aluno do curso de atualização em Dor Têmporo-Mandibular -

\section{Victor Quaglio}

Cirurgião-dentista graduado pela UFF e aluno do curso de Especializando em Implantodontia - USP

\section{Suelen Cristina Sartoretto}

Professora das disciplinas de Cirurgia Bucal e Anatomia da Universidade Iguaçu UNIG

\section{Marcelo José Uzeda}

Professor das disciplinas de Cirurgia Oral Menor e Anestesiologia da Universidade Federal Fluminense - UFF e Professor das disciplinas de Cirurgia Bucal e Anatomia da Universidade Iguaçu - UNIG

Instituição na qual o trabalho foi realizado: Universidade Federal Fluminense

Categoria: Revisão de Literatura

\section{Informações do autor principal:}

Farley Souza Cunha

Rua Mário Santos Braga, 28

Centro - Niterói - RJ, Brasil.

CEP: $24020-140$

E-mail: farleysouzac@gmail.com

Telefone: $+552196849-3677$ 
COPYRIGHT @ 2019 INTERNATIONAL JOURNAL OF SCIENCE DENTISTRY | AVAILABLE ONLINE

http://www.periodicos.uff.br/index

\section{RESUMO}

A Comunicação Buco-Sinusal (CBS) é um incidente cirúrgico que pode ocorrer durante a exodontia de dentes maxilares posteriores devido à intimidade de suas raízes com o assoalho do seio maxilar. As CBSs com diâmetro de até $2 \mathrm{~mm}$ tendem a curar-se sem tratamento específico. Entretanto, as maiores que $3 \mathrm{~mm}$ se não tratadas adequadamente, podem evoluir para complicações tais como sinusite maxilar recorrente, alteração do timbre nasal além da passagem de líquidos da cavidade bucal para a nasal através do óstio dentre outras. Este trabalho tem por objetivo discutir as técnicas mais utilizadas para o fechamento de CBS comparando-as com a técnica que utiliza o corpo adiposo bucal (Bola de Bichat), avaliando suas vantagens e desvantagens. As bases de dados digitais acessadas foram PubMed, MEDLINE, LILACS, Scopus compreendendo artigos em inglês entre o período de 1988 a 2016. Concluímos que o uso desta técnica apresenta excelentes resultados com poucas complicações, restituindo ao paciente uma condição de normalidade.

Palavras-Chave: Fístula Bucoantral, Fístula Bucosinusal, Corpo adiposo bucal, bola de Bichat, Comunicação Bucosinusal.

\section{ABSTRACT}

Oroantral Communication (OC) is a surgical incident that can occur during posterior maxillary teeth extraction due to the anatomical intimacy of its roots with the lining of the maxillary sinus. OC up to $2 \mathrm{~mm}$ in diameter can cure without specific treatment. However, as larger than $3 \mathrm{~mm}$ if left untreated, they may progress to complications such as recurrent maximal sinusitis, alteration of the nasal timbre beyond the passage of the oral cavity to a nasal through the ostium, among others. Several surgical techniques have been developed for the treatment of OC. This paper aims to review the literature on how to evaluate the most commonly used techniques for closing the $\mathrm{OC}$, comparing them with a technique that uses the buccal adipose body (Bichat Ball), discussing its advantages and advantages. The digital databases accessed were PubMed, MEDLINE, LILACS and Scopus comprising articles in English from 1988 to 2016. This study allows us to conclude that the use of this technique presents excellent results with few complications, restoring the patient to a normal condition.

Key-Words: Oroantral fistula, Oroantral communication, buccal fat pad, bichat fat pad 
COPYRIGHT @ 2019 INTERNATIONAL JOURNAL OF SCIENCE DENTISTRY | AVAILABLE ONLINE

http://www.periodicos.uff.br/index

\section{INTRODUÇÃO}

A anatomia do seio maxilar é conhecida por apresentar o seu assoalho delgado e proximidade com dentes posteriores da maxila. Procedimentos cirúrgicos para extração de molares superiores, fraturas do tipo Le Fort I, trauma direto, infecções crônicas, problemas na cicatrização após realizada uma incisão do tipo Caldwell-luc, osteomielite da maxila, sífilis, radioterapia, ressecção de cisto ou tumor próximo à região, entre outras podem causar uma comunicação da cavidade bucal com o seio maxilar, caracterizando assim uma comunicação buco-sinusal (GEORGIADE N, et al. 1969; AL-JUBOORI MJ, et al. 2018).

Uma comunicação buco-sinusal tem como sintomas dor localizada, escape de ar da cavidade nasal para a cavidade oral, resultando em voz anasalada, mau-cheiro, secreção purulenta e rinorréia purulenta pelo lado afetado. Essa comunicação pode evoluir clínica e patologicamente para uma fístula buco-sinusal que, se não tratada pode gerar diversas complicações como osteomielite, sinusopatias e todas as complicações relacionadas a essas doenças. Comunicações com menos de $2 \mathrm{~mm}$ comumente cicatrizam espontaneamente não exigindo cuidados cirúrgicos especiais além de acompanhamento clínico regular e boa higienização do local mas, aquelas com diâmetro maior que $3 \mathrm{~mm}$ ou associadas a doença periodontal ou infecção devem ser fechadas cirurgicamente. Egyedi em 1977 foi o primeiro a utilizar o tecido adiposo jugal como enxerto pediculado em cirurgia de fechamento de comunicação buco-sinusal, abrindo caminho para que outros pesquisassem acerca do assunto, ampliando o que é sabido sobre essa cirurgia até os dias de hoje (AWANG M, 1988).

Para o fechamento, diversas técnicas vêm sido descritas na literatura, como retalhos deslizantes vestibulares, retalhos rotatórios palatinos, enxertos osséos, com discos de ouro de 24 quilates e uso da bola de Bichat (EEH I, MEF A, 1999; ALJUBOORI MJ, et al. 2018). O presente trabalho tem por objetivo revisar a literatura a fim de avaliar os resultados e complicações da técnica de fechamento de FBS com a utilização do coxim adiposo jugal, fornecendo embasamento teórico para que os cirurgiões dentistas sejam capazes de realizar esse procedimento e esclarecer os resultados obtidos quando utilizado. 
COPYRIGHT @ 2019 INTERNATIONAL JOURNAL OF SCIENCE DENTISTRY | AVAILABLE ONLINE

http://www.periodicos.uff.br/index

\section{MATERIAIS E MÉTODO}

A pesquisa foi realizada através das bases de dados digitais PubMed, MEDLINE, LILACS, Scopus compreendendo artigos em inglês de 1988 a 2018 acrescentando-se os trabalhos clássicos acerca do assunto, sendo encontrados 72 artigos e selecionados ensaios clínicos de pesquisa contendo mais de 10 pacientes e mais citados, sendo selecionados 19 artigos. As palavras-chave usadas foram Fístula Bucosinusal, Comunicação Bucosinusal, Corpo Adiposo Bucal, Bola de Bichat, Coxim Adiposo Jugal e Fístula Bucoantral.

\section{REVISÃO DA LITERATURA}

O tecido adiposo bucal foi descrito pela primeira vez por Heister em 1732, que acreditou que essa estrutura seria uma glândula, nomeando-a de "glândula molaris". Em 1802, Bichat descobriu sua verdadeira natureza: uma bola de gordura, que recebeu o nome de bola de Bichat. Entretanto, somente no início do século XX, a anatomia da estrutura foi publicada por Scammon, em 1919, sendo complementada por Gaughran em 1957 (EGYEDI P, 1977). Em 1977, Egyedi foi o primeiro a utilizar a bola de Bichat como um enxerto pediculado em fechamento de comunicações com diametros entre 1 e $4 \mathrm{~cm}$, o que estimulou importantes estudos tais como o trabalho de Neder, que descreveu o uso do coxim adiposo jugal como um enxerto livre para fechamento de defeitos orais e o de Tideman et al, que descreveu em seu estudo a anatomia detalhada da estrutura, incluindo a vascularização e a técnica operatória (TIDEMAN H, et al. 1986). Desde então, diversas técnicas e relatos de caso têm sido publicados incluindo o uso do plasma rico em fibrina (PRF) acerca do assunto (AL-JUBOORI MJ, et al. 2018).

O coxim adiposo jugal é uma massa lobulada simples com um corpo central e quatro extensões: bucal, pterigóidea, pterigopalatina e temporal. No adulto, o corpo principal encontra-se junto ao periósteo, que reveste a porção posterior da maxila, limitando-se na fossa pterigopalatina, musculo bucinador e masseter. O ducto de Stenon ou parotídeo atravessa a bola de Bichat na região anterior à glândula parótida em sentido 
COPYRIGHT @ 2019 INTERNATIONAL JOURNAL OF SCIENCE DENTISTRY | AVAILABLE ONLINE

http://www.periodicos.uff.br/index

medial, emergindo na cavidade oral. A extensão bucal do coxim adiposo repousa sobre a fáscia bucofaríngea, localizada na superfície externa do músculo bucinador, contribuindo, assim, para o contorno da bochecha. A soma das massas do corpo principal e da extensão bucal resultam entre $55 \%$ - 70\% do total de massa do tecido adiposo bucal. A extensão pterigopalatina segue anteromedialmente ao corpo principal, chegando até o forame redondo junto ao nervo maxilar. Outra extensão, a temporal, passa superiormente ao arco zigomático e divide-se em duas porções: a superficial, que passa supero-posteriormente entre a fáscia temporal e a superfície do músculo temporal, e a rofunda, que passa entre as fibras profundas do músculo temporal até a asa maior do osso esfenóide. Cada porção da extensão temporal tem sua própria cápsula, estando ancoradas em estruturas circundantes por ligamentos, sendo diferentes anatomicamente e vascularmente do restante do corpo adiposo jugal. Por fim, a extensão pterigóidea se restringe ao espaço pterigomandibular, medialmente ao músculo pterigóideo medial, envolvendo tanto os nervos e vasos alveolares inferiores, como o nervo lingual, e indo até a região anterior da porção profunda da glândula parótida. A irrigação da bola de Bichat é dada pelos ramos bucais, da artéria temporal profunda, ramos da artéria facial e do ramo transverso da face da temporal superficial (GAUGHRAN GR, 1957; VAUGHAN E, BROWN A, 1983; HANAZAWA Y, 1995).

A Comunicação buco-sinusal (CBS) é uma condição caracterizada por uma abertura entre a cavidade oral e o seio maxilar. Tal condição pode ser causada por extração de molares superiores, fraturas do tipo Le Fort I, trauma direto, lesões inflamatórias agudas ou crônicas próximas das raízes dos molares superiores, osteomielite da maxila, sífilis, entre outras, podendo apresentar fechamento espontâneo ou evoluir clínica e histologicamente para uma fístula epitelizada (COULTHARD P, et al. 2013). CBS menores do que $2 \mathrm{~mm}$ em seu maior diâmetro, tendem a se fechar espontaneamente, sendo necessário somente o acompanhamento clínico e uma boa higienização do local. Aquelas com diâmetro maior do que $3 \mathrm{~mm}$ ou as associadas à sinusite do seio maxilar ou doença periodontal, podem persistir, sendo indicada, então, intervenção cirúrgica para o tratamento (ABUABARA A, et al.2006). A queixa principal de pacientes com CBS é a saída de bebida ou outros alimentos pelo nariz ao ingerir líquidos. Outros sinais comuns são sinusites recorrentes, voz anasalada, mau hálito, tosse noturna, paladar alterado, coriza, desaparecimento de coágulo nos primeiros dias após a extração de molar, fratura 
COPYRIGHT @ 2019 INTERNATIONAL JOURNAL OF SCIENCE DENTISTRY | AVAILABLE ONLINE

http://www.periodicos.uff.br/index

do soalho de seio maxilar e obstrução nasal do lado afetado. Se a CBS não for tratada, algumas complicações mais graves podem surgir, como por exemplo, candidíase, infecção crônica do seio maxilar e até osteomielite (JADHAV KKB, et al. 2014). O diagnóstico de uma comunicação buco-sinusal pode ser realizado através do exame clínico, observando-se a presença de tecido de granulação no alvéolo do elemento extraído, além da execução de manobra de valsava, quando se pede ao paciente para soprar suavemente o ar pelo nariz tampado estando com a boca aberta, podendo ser observada a passagem de ar ou formação de bolhas na eventual comunicação. $\mathrm{O}$ exame radiográfico e a tomografia computadorizada complementam o diagnóstico indicando tamanho e localização da fístula, além de evidenciar uma possível presença de sinusite ou a presença de corpos estranhos intra-sinusais (Figura 1 e 2).

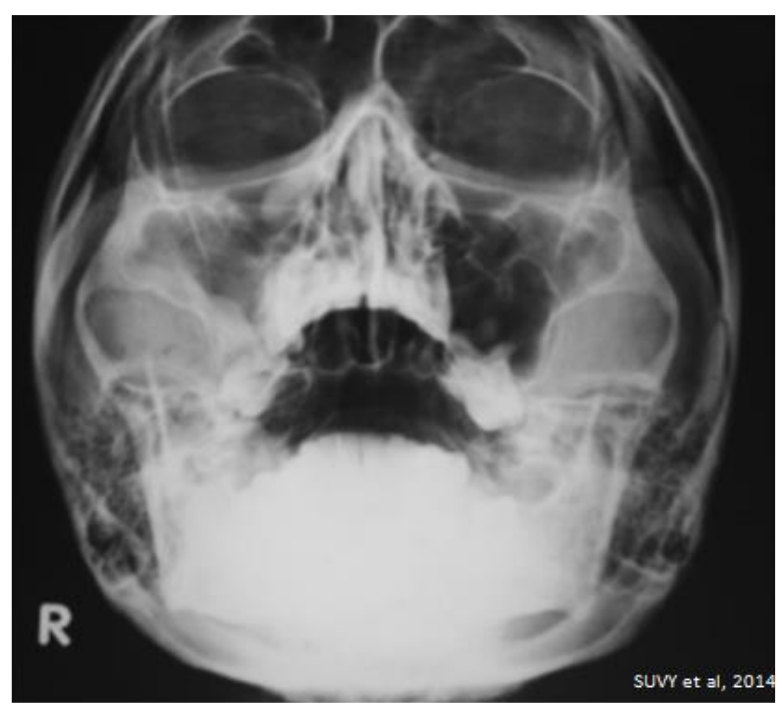

Figura 1 - Radiografia na incidência de Waters mostrando a radiopacidade sugestiva de sinusite maxilar direita (SUVY M, et al. 2015)

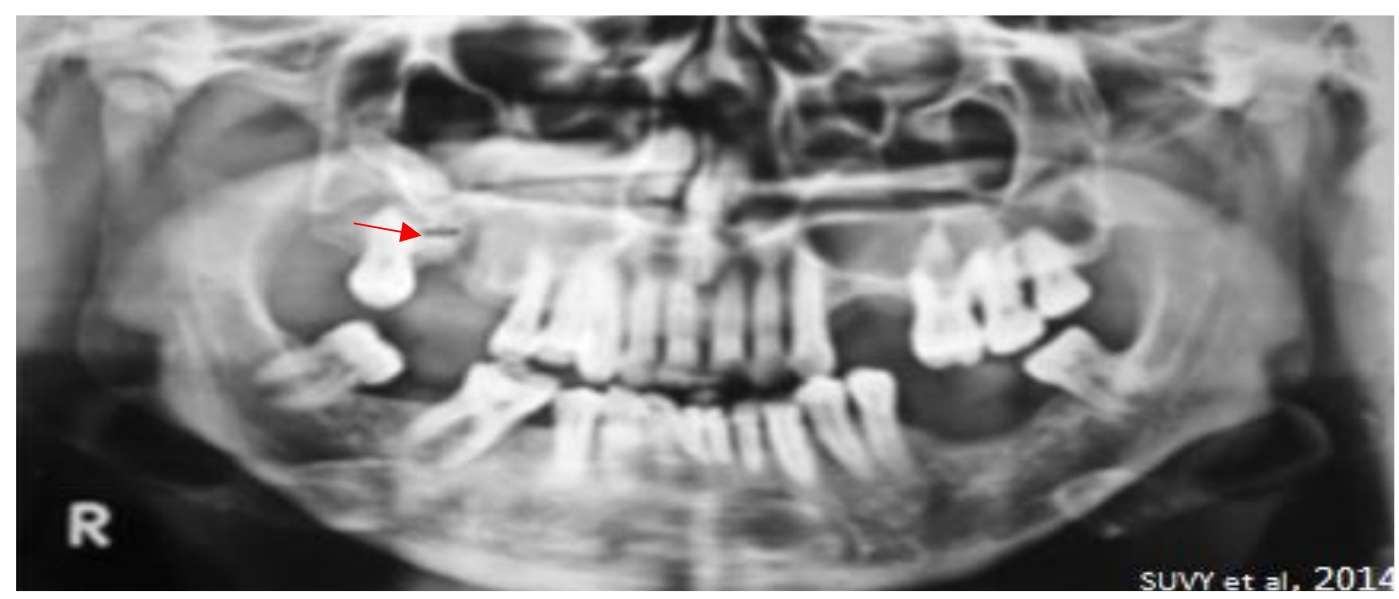

Figura 2 - Radiografia panorâmica sugerindo uma comunicação bucosinusal na região do primeiro molar superior direito (SUVY M, et al. 2015) 
COPYRIGHT @ 2019 INTERNATIONAL JOURNAL OF SCIENCE DENTISTRY | AVAILABLE ONLINE

http://www.periodicos.uff.br/index

O tratamento da CBS tem como objetivo selar essa abertura e promover um crescimento ósseo na região. Diversas técnicas vêm sendo desenvolvidas para atingir esse objetivo, entre elas destacam-se: o uso de retalhos locais, como o retalho vestibular, o retalho palatino ou retalho duplo: vestibular e palatino. No entanto, esses procedimentos resultam em grandes áreas cruentas e uma diminuição do sulco vestibular. Além disso, mostram-se pouco eficazes para corrigir grandes defeitos (EEH I, MEF A, 1999; ZIDE MF, KARAS ND, 1992). O uso de outros tipos de retalhos como os retalhos baseados em língua e retalhos nasolabiais também já foram utilizados, assim como o uso de osso trabeculado ou de materiais aloplásticos, como polimetil metracrilato, além do uso de PRF associado ou não a biomateriais (AL-SIBAHI A, SHANOON A, 1982; AlJUBOORI, et al. 2018). O uso do tecido adiposo bucal vem ultimamente sendo bastante estudado apresentando-se de fácil técnica cirúrgica e com resultados bastante promissores (ABUABARA, A. et al. 2006, SUVY M, et al. 2015) Após realizar os procedimentos de anestesia local ou geral deve-se excisar a região fistulada com uma lâmina de bisturi e curetar a região até remoção de todo o tecido de granulação presente, em seguida, devese avivar os bordos da fístula. Para coleta da bola de Bichat deve-se realizar uma incisão mucoperiostal em região posterior do pilar zigomático-maxilar até romper a fascia do corpo adiposo. Com uma pinça hemostática curva ou tesoura de metzembaum realizar a dissecção suavemente da região ântero-medial do processo coronóide da mandíbula até expor a gordura bucal amarelada. O tecido adiposo é então delicadamente removido enquanto se disseca as estruturas circundantes da bola. O uso de sugador deve ser evitado pelo risco de aspiração devido à consistência do tecido (JASMEET SKP, RAGANATH $K$, 2010). A bola de Bichat deve ser levada até a região da comunicação ou fístula e ser posicionada de modo a vedar a comunicação, sendo suturada em posição com cuidado para não romper a capsula do corpo adiposo (HANAZAWA Y, 1995). O uso de retalhos vestibulares e/ou palatinos podem ser feitos a partir deste momento, assim como o uso de membranas. Deve-se acompanhar o paciente por ao menos uma semana e garantir que todas as orientações estejam sendo seguidas. Clinicamente a superfície exposta da bola fica esbranquiçada em 3 dias após o procedimento e apresenta-se gradualmente vermelha até 1 semana devido a formação de tecido de granulação, para que até a terceira semana, se torne completamente epitelizada (Figura 3). 


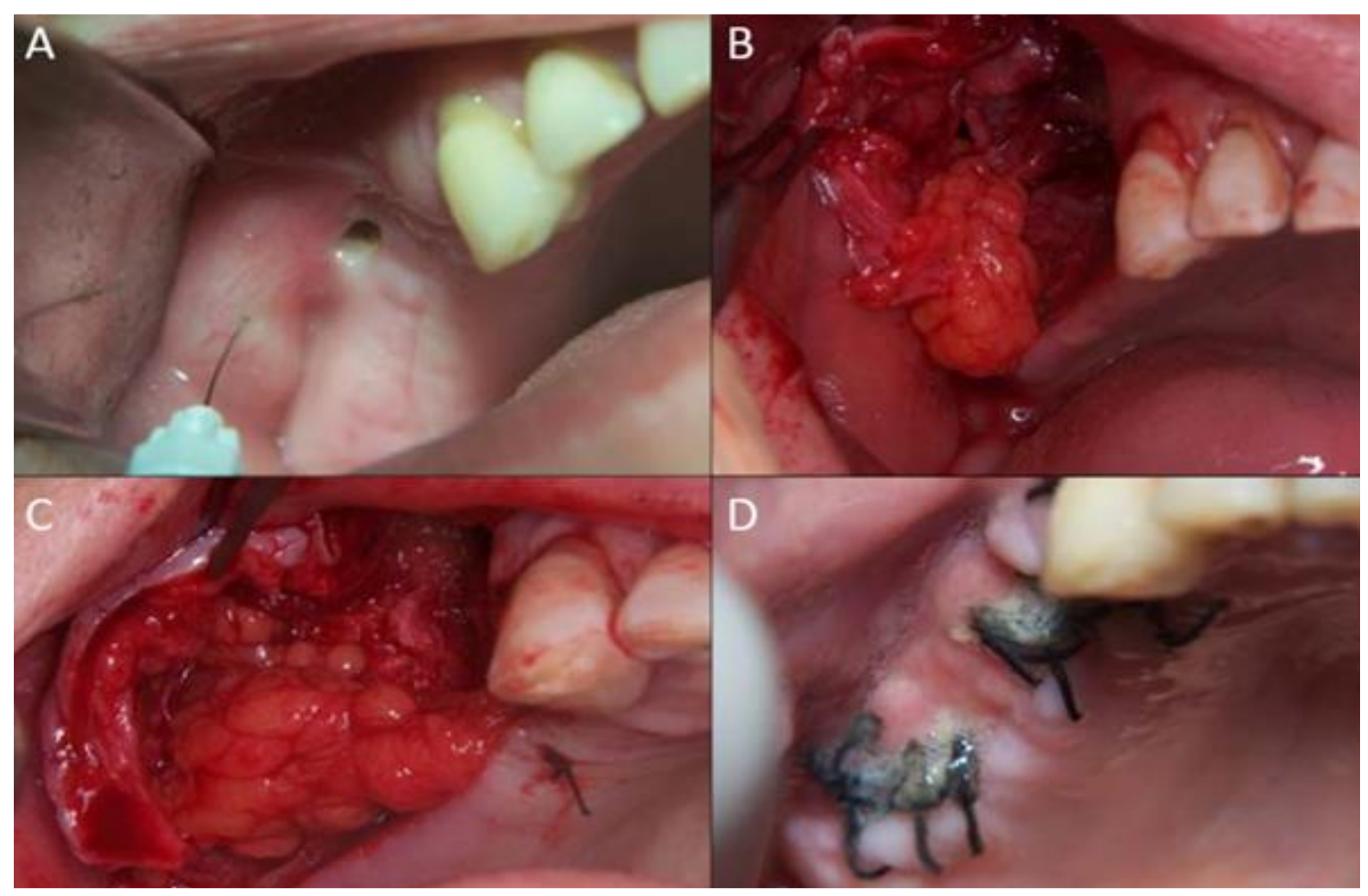

Figura 3: Uso da bola de Bichat para fechamento de CBS: A) Fístula em região do primeiro molar superior direito, B) Defeito ósseo e obtenção do coxim adiposo, C) Posicionamento do coxim adiposo sobre o defeito, D) Aspecto pós-operatório de sete dias (ABAD-GALLEGOS M, 2011).

\section{DISCUSSÃO}

A literatura revisada permite observar concordância entre os autores quanto à utilização da bola de Bichat para fechamento de comunicações e fístulas buco-sinusais. Isso é demonstrado pelo alto índice de sucesso, entre $92,5 \%$ e $100 \%$ obtido por todos os autores analisados nesta revisão. A grande vascularização presente no coxim gorduroso pode explicar o esse sucesso da técnica e a rápida epitelização da gordura, provada histológicamente pela migração de células epiteliais estratificadas escamosas da mucosa adjacente. $\mathrm{O}$ volume da bola de Bichat varia entre $8,33 \mathrm{ml}$ e $11,9 \mathrm{ml}$, tamanho bem constante entre os indivíduos e pouco alterada de acordo com o peso e distribuição da gordura de um indivíduo permite a disponobilidade suficiente de tecido para o emprego da técnica (JASMEET SKP, RAGANATH K, 2010). Dos pacientes nos quais o procedimento falhou, todos apresentaram fístula recorrente e necessitaram de uma nova cirurgia. Entre as intercorrências relatadas, podemos destacar a hipertrofia da mucosa associada ao excesso de tecido de granulação em 9 pacientes, rinite persistente em 2 
COPYRIGHT @ 2019 INTERNATIONAL JOURNAL OF SCIENCE DENTISTRY | AVAILABLE ONLINE

http://www.periodicos.uff.br/index

pacientes e sinéquia da mucosa em 1 paciente(DOLANMAZ D, et al. 2004; HOROWITZ $\mathrm{G}$, et al. 2016). Outras complicações podem incluir o hematoma, a necrose total ou parcial do enxerto adiposo, além de infecção deste e a injuria do nervo facial quando há tracionamento intenso da bola de Bichat (ABUABARA A, et al. 2006; TIDEMAN H, et al. 1986). Ao compararmos a técnica do fechamento das comunicações buco-sinusais com bola de Bichat com a técnica que emprega o uso dos retalhos vestibulares observamos que este apresenta em alguns casos uma perfusão sanguínea deficiente quando em grandes volumes, além do estreitamento do fundo de vestíbulo, não sendo recomendadas para grandes defeitos, diferentemente do uso do tecido adiposo jugal, que apresenta excelentes resultados em grandes defeitos dispensando o uso de retalhos vestibulares extensos ((ABUABARA A, et al. 2006; EEH I, MEF A, 1999; HOROWITZ G, et al.2016). Em relação aos retalhos palatinos estes apresentam regenerações mais lentas devido à região doadora, demorando até 3 semanas a mais para a cicatrização aumentando o desconforto pós-operatório (EEH I, MEF A, 1999). Uma alternativa recente, que muito vem encantando a comunidade científica, é o uso dos agregados plaquetários para o tratamento da CBS que tem demostrado ótimo resultado devido a rápida epitelização e ausência de recidivas (AL-JUBOORI MJ, et al. 2018).

\section{CONCLUSÃO}

Este trabalho nos permite concluir que a técnica cirúrgica para fechamento de comunicação buco-sinusal através da utilização da bola gordurosa de Bichat é uma ótima alternativa as demais técnicas usualmente empregadas para tal, obliterando a comunicação da cavidade oral com o seio maxilar de forma eficiente e permitindo um pós-operatório menos desconfortável para o paciente que mais rapidamente poderá retomar sua condição de normalidade. 
COPYRIGHT @ 2019 INTERNATIONAL JOURNAL OF SCIENCE DENTISTRY | AVAILABLE ONLINE

http://www.periodicos.uff.br/index

\section{REFERÊNCIAS BIBLIOGRÁFICAS}

1. GEORGIADE N, MALDICK R, THOME F. The nasolabial tunnel flap. Plastic and Reconstructive Surgery 1969, 43: 463-65.

2. AL-JUBOORI MJ, AL-ATTAS MA, FILHO LCM. Treatment of chronic oroantral fistula with platelet-rich fibrin clot and collagen membrane: a case report. Clinical, Cosmetic and Investigational Dentistry 2018:10 245-249

3. AWANG M. Closure of oroantral fistula. International Journal of Oral and Maxillofacial Surgery. 1988.

4. EEH I, MEF A. The use of the pedicled buccal fat pad (BFP) and palatal rotating flaps in closure of oroantral communication and palatal defects. The Journal of Laryngology and Otology. September 1999, Vol. 113, pp. 834-838.

5. EGYEDI, P. Utilization of the buccal fat pad for closure of oroantral and/or oronasal communication. J Maxillofac Surg;1977, 5:241

6. TIDEMAN H, BOSDNQUET A, SCOTT J. Use of the buccal fat pad as a pedicled graft. J Oral Maxillofac Surg 1986;44:435-40.

7. GAUGHRAN GR: Fasciae of the masticator space. Anat Rec 129:383, 1957

8. VAUGHAN E, BROWN A. The versatility of the lateral tongue flap in the reconstruction of defects of the oral cavity. British Journal of Oral Surgery 1983, 21: 1-4.

9. HANAZAWA Y. Closure of Oroantral Communications Using a Pedicled Buccal Fat Pad Graft. J Oral Maxillofac Surg; 1995, 53:771-775.

10. COUlthard, P, et al. Master Dentistry. 3rd ed. Churchill Livingstone, 2013. Print.

11. ABUABARA, A. et al. Evaluation of different treatments for oroantral/oronoasal communications: experience of 112 cases. International Journal of Oral \& Maxillofacial Surgery. Elsevier Ltd. 35:155-158. 2006.

12. JADHAV KKB, MUJIB BRA, GUPTA N. Cytological Approach for Diagnoses of Non-healing Oroantral Fistula Associated with Candidiasis. Journal of Cytology. 31 1: 47-9in "Journal of Cytology” 31:47 a 49. 2014.

13. SUVY M, SUREJ K, PARVATHI RN. The Versatility in the Use of Buccal Fat Pad in the Closure of Oro-antral Fistulas. J Maxillofac Oral Surg. 2015, 14: 374 
COPYRIGHT @ 2019 INTERNATIONAL JOURNAL OF SCIENCE DENTISTRY | AVAILABLE ONLINE http://www.periodicos.uff.br/index

14. ZIDE MF, KARAS ND. Hydroxylapatite block closure of oroantral fistulas: report of cases. Journal of Oral and Maxillofacial Surgery 1992, 50: 71-75.

15. AL-SIBAHI A, SHANOON A. The use of soft polymethylmethacrylate in the closure of oroantral fistula. Journal of Oral and Maxillofacial Surgery 1982, 40: 165-166.

16. JASMEET SKP, RANGANATH K. Buccal pad of fat and its applications in oral and maxillofacial surgery: a review of published literature (February) 2004 to (July) 2009. Oral Surg Oral Med Oral Pathol Oral Radiol Endod 2010;110:698-705.

17. ABAD-GALLEGOS M. Use of Bichat's buccal fat pad for the sealing of orosinusal communications. A presentation of 8 cases. Med Oral Patol Oral Cir Bucal. 2011 Mar 1;16 2:e214-8.

18. DOLANMAZ D, TUZ H, BAYRAKTAR S, METIN M, ERDEM E, BAYKUL T. Use of pedicled buccal fat pad in the closure of oroantral communication: analysis of 75 cases. Quintessence Int. 2004 Mar;35(3):241-6.

19. HOROWITZ G, KOREN I, CARMEL NN, BALABAN S, ABU-GHANEM S, FLISS DM, et al. One stage combined endoscopic and per-oral buccal fat pad approach for large oro-antral-fistula closure with secondary chronic maxillary sinusitis. Eur Arch Otorhinolaryngol. 2016 Apr;273(4):905-9. 